\title{
Differential expression and predictive value of monocyte scavenger receptor CD163 in populations with different tuberculosis infection statuses
}

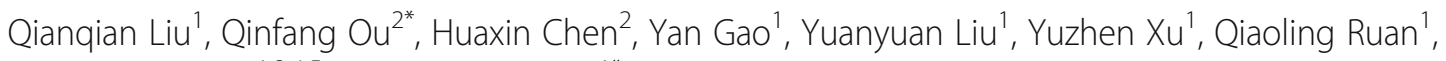
Wenhong Zhang ${ }^{1,3,4,5}$ and Lingyun Shao ${ }^{1 *}$ (D)

\begin{abstract}
Background: Monocytes are the predominant innate immune cells at the early stage of Mycobacterium tuberculosis (M. tb) infection as the host defense against intracellular pathogens. Understanding the profile of different monocyte subpopulations and the dynamics of monocyte-related biomarkers may be useful for the diagnosis and prognosis of tuberculosis.

Methods: We enrolled 129 individuals comprising patients with pulmonary tuberculosis (PTB) $(n=39)$, tuberculous pleurisy (TBP) $(n=28)$, malignant pleural effusion (MPE) $(n=21)$, latent tuberculosis infection (LTBI) $(n=20)$, and healthy controls $(H C)(n=21)$. Surface expression of CD14, CD16, and CD163 on monocytes was detected using flow cytometry. In addition, soluble CD163 (sCD163) was determined by enzyme linked immunosorbent assay.

Results: Higher frequency of $\mathrm{CD}_{1}{ }^{+} \mathrm{CD}_{16} 6^{+}(15.7 \%$ vs $7.8 \%, P<0.0001)$ and $\mathrm{CD} 14^{-} \mathrm{CD} 16^{+}(5.3 \%$ vs $2.5 \%, P=0.0011)$ monocytes and a decreased percentage of $\mathrm{CD}_{14}{ }^{+} \mathrm{CD} 16^{-}(51.0 \%$ vs $70.4 \%, P=0.0110)$ cells was observed in PTB patients than in HCs. Moreover, PTB patients displayed a higher frequency of $\mathrm{CD} 163^{+}$cells in $\mathrm{CD} 16^{+}$monocytes than those in the HC group $(40.4 \%$ vs $11.3 \%, P<0.0001)$. The level of SCD163 was elevated in TBP patients and was higher in pleural effusion than in plasma $(2116.0 \mathrm{ng} / \mathrm{ml}$ vs $1236.0 \mathrm{ng} / \mathrm{ml}, P<0.0001)$. sCD163 levels in pleural effusion and plasma could be used to distinguish TBP from MPE patients (cut-off values: 1950.0 and $934.7 \mathrm{ng} / \mathrm{ml}$, respectively; AUCs: 0.8418 and 0.8136 , respectively). Importantly, plasma sCD163 levels in TBP patients decreased significantly after anti-TB treatment.
\end{abstract}

Conclusions: Higher expression of membrane and soluble CD163 in active tuberculosis patients might provide insights regarding the pathogenesis of tuberculosis, and SCD163 may be a novel biomarker to distinguish TBP from MPE and to predict disease severity.

Keywords: Tuberculosis, Monocyte subpopulations, CD163, Innate immunity, Disease severity

\footnotetext{
*Correspondence: oqinfang@163.com; lingyun26@fudan.edu.cn

${ }^{2}$ Department of Pulmonary Diseases, Wuxi Infectious Diseases Hospital, Wuxi

214005, China

'Department of Infectious Diseases, Huashan Hospital, Fudan University, 12

Wulumuqi Zhong Road, Shanghai 200040, China

Full list of author information is available at the end of the article
}

(c) The Author(s). 2019 Open Access This article is distributed under the terms of the Creative Commons Attribution 4.0 International License (http://creativecommons.org/licenses/by/4.0/), which permits unrestricted use, distribution, and reproduction in any medium, provided you give appropriate credit to the original author(s) and the source, provide a link to the Creative Commons license, and indicate if changes were made. The Creative Commons Public Domain Dedication waiver (http://creativecommons.org/publicdomain/zero/1.0/) applies to the data made available in this article, unless otherwise stated. 


\section{Background}

Mycobacterium tuberculosis ( $M . t b)$ invades the host and activates immune responses including innate and adaptive immunity, and causes tuberculosis (TB), which remains a global public health concerns [1]. Monocytes/ macrophages, as the first line of defense, are critical for host immunity against $M$. tb infection [2-4]. Human monocytes are classified into three major subpopulations based on the expression of markers CD14 and CD16: $\mathrm{CD} 14^{+} \mathrm{CD} 16^{-}$(classical), $\mathrm{CD} 14^{+} \mathrm{CD} 16^{+}$(intermediate), and $\mathrm{CD} 14^{-} \mathrm{CD} 16^{+}$(non-classical) monocytes [5-7]. The three monocyte subsets represent different stages of macrophage differentiation and play different roles in $M$. $t b$ infection [8]. Classical monocytes account for the majority of total subsets; they differentiate into proinflammatory M1 macrophages (classical activated) that are permissive to $M$. $t b$ infection in vitro and produce several pro-inflammatory cytokines [8]. However, both non-classical and intermediate monocytes are considered precursors of anti-inflammatory M2 macrophages (alternative activated) in different disease conditions [8].

Increasing evidence suggests that switching the M1/ M2 phenotype influences the clinical outcome of host infection with $M . t b[2,3,9,10]$. The early stage of anti-TB immune responses is predominated by M1 macrophages, which are characterized by high production of iNOS and IFN- $\gamma$, with the function of killing most $M$. $t b$ and restricting the replication of the remainder. Nevertheless, M2 macrophages are poorly microbicidal and play an immunomodulatory role [3]. Thus, the shift from M1 to M2 polarization during $M$. $t b$ infection might be a microbial strategy to escape immune attack and cause disease progression.

CD163, a scavenger receptor that serves as an M2 macrophage phenotype marker, is also expressed on monocytes, and binds to haptoglobin-hemoglobin complexes, mediating their endocytosis [11]. In the context of TB, expansion of $\mathrm{CD}_{16}{ }^{+} \mathrm{CD} 163^{+} \mathrm{MerTK}^{+}$monocytes contribute negatively to the host defense against $M$. $t b$ by a low ratio of pro-/anti-inflammatory cytokine production and a poor capacity to activate T cells. Moreover, CD163 and MerTK act as M2-like macrophage activation markers, which are characterized by pathogen permissivity and immunomodulatory activity [12]. Indeed, the soluble form of CD163 (sCD163) from monocyte activation, exists in plasma and is correlated with TB disease severity. In this study, we explored the expression of scavenger receptor CD163 on monocyte subsets in populations with different tuberculosis infection statuses including active tuberculosis, latent tuberculosis infection and non-infection, detected the sCD163 levels in plasma and pleural effusions, further assessed the value of sCD163 in diagnosing tuberculosis and in predicting the disease severity and treatment outcome.

\section{Methods}

\section{Study population}

In total, 129 individuals were enrolled in this study including patients with pulmonary TB (PTB) $(n=39)$, tuberculous pleurisy (TBP) $(n=28)$, malignant pleural effusion (MPE) $(n=21)$, latent tuberculosis infection (LTBI) $(n=20)$, and healthy controls $(\mathrm{HC})(n=21)$. All the patients were recruited from Wuxi Fifth People's Hospital, Zhuji People's Hospital, and Fudan University Affiliated Huashan Hospital from 2011 to 2018. Populations with LTBI and HC were recruited from the relatives of PTB patients and the volunteers of Huashan Hospital during the same period.

This study was approved by the Ethics committee of Huashan Hospital, Fudan University. Written informed consent was obtained from all the participants.

\section{Diagnosis criteria}

PTB patients were diagnosed based on identification of $M$. $t b$ in the sputum or bronchoscopy. TBP patients were diagnosed according to the following criteria: 1 ) pleural biopsy; 2) acid-fast bacilli (AFB) smear or culture positive in pleural effusion (PE); 3) AFB smear or culture positive in sputum; 4) combination with clinical symptoms, radiological results, and effective anti-TB treatment upon lack of etiology evidence. MPE patients without tuberculosis infection were enrolled as controls, diagnosed based on either histopathology in pleural tissue or cytology in PE. Populations with LTBI and HC were interferon- $\gamma$ release assay (IGRA)-positive and -negative, respectively. In addition, they had no evidence of active tuberculosis infection.

All enrolled participants were free of HIV infection, autoimmune disease or other chronic infections (i.e., chronic HBV/HCV infection). Furthermore, they were not undergoing immune-modulating treatment.

\section{Interferon- $\gamma$ release assay (IGRA)}

In this study, the T-cell-based enzyme-linked immunospot assay for tuberculosis (T-SPOT.TB) (Oxford Immunote Ltd., Oxford, UK) was performed as IGRA according to the manufacturer's instructions. The positive results were analyzed as described previously [13].

\section{Cell surface staining and flow cytometry}

Peripheral blood mononuclear cells (PBMCs) were separated by Ficoll density-gradient centrifugation from $10 \mathrm{ml}$ venous blood samples. For phenotyping of monocyte subsets, one million fresh PBMCs were surface stained with monoclonal anti-human CD14-APC (61D3, eBioscience), CD16-FITC (3G8, Biolegend) and CD163-BV421 (GHI/ 61, Biolegend) at room temperature in the dark for 15 min, and were washed twice in PBS containing 2\% FBS. Stained samples were detected on a Beckman Moflo flow 
cytometer. According to the expression of CD14 and CD16, three major monocyte subsets in PTB patients and controls were analyzed as classical $\left(\mathrm{CD} 14^{+} \mathrm{CD} 16^{-}\right)$, intermediate $\left(\mathrm{CD} 14^{+} \mathrm{CD} 16^{+}\right)$, and non-classical $\left(\mathrm{CD} 14^{-} \mathrm{CD} 16^{+}\right)$ monocytes. Furthermore, expression of the scavenger receptor CD163 on monocytes subsets was evaluated. Data were analyzed using Flowjo 10 (Tree Star, Inc. Ashland, OR).

\section{Detection of soluble CD163 levels using enzyme linked immunosorbent assay (ELISA)}

Levels of soluble (s)CD163 were assessed in plasma and pleural effusion (PE) samples stored at $-80{ }^{\circ} \mathrm{C}$ using an ELISA kit (DC1630, R\&D systems) according to the manufacture's protocol. Absorbance was immediately determined at $450 \mathrm{~nm}$ and $570 \mathrm{~nm}$ on a microplate reader, and was corrected by subtracting the readings at $570 \mathrm{~nm}$ from the readings at $450 \mathrm{~nm}$. The minimum detectable dose was $0.177 \mathrm{ng} / \mathrm{ml}$.

\section{Statistical analysis}

Statistical analysis was performed using GraphPad Prism 6 (GraphPad Software, Inc. La Jolla, CA) and MultiExperiment Viewer (MeV) 4.9 (Dana-Farber Cancer Institute, Boston, USA). Two different groups were compared using an unpaired $t$ test or Mann-Whitney $U$ test (when the variances were significantly different). Categorical variables were compared using the $\chi^{2}$ test or Fisher's exact test, as appropriate. Receiver operating characteristics (ROC) curve analysis was performed to evaluate the diagnostic performance of sCD163 levels in plasma or the PE for differential diagnosis of PTB and controls, or TBP and MPE. Comparisons of plasma sCD163 levels in unpaired pre- and post-treatment groups were performed using unpaired Mann-Whitney $U$ test. Statistical significance was referred as $P<0.05$.

\section{Results}

\section{Clinical characteristics of participants}

The 129 enrolled individuals were divided into five groups, as shown in Table 1. Of the PTB group $(n=39)$, 35 confirmed cases based on sputum AFB smear positive or culture positive for $M$. $t b$, and 4 patients diagnosed by bronchoscopy were included. Among 28 patients with TBP, more than one quarter $(28.6 \%$ ) had pulmonary TB simultaneously, and 11 (39.3\%) were confirmed by pleural biopsy $(n=8)$ or by identification of AFB in pleural effusions $(n=3)$. In this study, nearly half of the active tuberculosis (ATB) patients $(n=31,46.3 \%)$ were anti-TB treatment naive at recruitment. The characteristics of all individuals are presented in Table 1.

\section{Increased percentage of circulating $\mathrm{CD} 16^{+}$monocytes and lack of classical (CD14 $\left.{ }^{+} \mathrm{CD} 16^{-}\right)$monocytes in active tuberculosis patients}

Given the importance of monocytes in the innate immune response to tuberculosis, we investigated the profiles of monocyte subsets in ATB patients based on the expression of CD14 and CD16. An evidently high expression of CD16 on monocytes was observed in the ATB group (Fig. 1b) compared to HC (Fig. 1c) $(22.0 \%$ vs $11.4 \%, P<$ 0.0001, Fig. 1d). Furthermore, ATB patients exhibited

Table 1 Clinical characteristics of enrolled individuals

\begin{tabular}{|c|c|c|c|c|c|c|}
\hline & \multicolumn{2}{|l|}{$\operatorname{ATB}(n=67)$} & \multirow{2}{*}{$\begin{array}{l}\text { MPE } \\
(n=21)\end{array}$} & \multirow{2}{*}{$\begin{array}{l}\text { LTBI } \\
(n=20)\end{array}$} & \multirow{2}{*}{$\begin{array}{l}\mathrm{HC} \\
(n=21)\end{array}$} & \multirow[t]{2}{*}{$P$ value } \\
\hline & $\mathrm{PTB}(n=39)$ & $\operatorname{TBP}(n=28)$ & & & & \\
\hline Male/Female & $30 / 9$ & $23 / 5$ & $16 / 5$ & $10 / 10$ & $13 / 8$ & 0.0992 \\
\hline Age, median (IQR) & $44(28-62)$ & $48(23-66)$ & $72(63-78)$ & $53(40-64)$ & $46(27-65)$ & $<0.0001$ \\
\hline History of BCG vaccination, n (\%) & $24(61.5)$ & $19(67.8)$ & $11(52.4)$ & $15(75.0)$ & $17(80.9)$ & 0.2913 \\
\hline IGRA+, n (\%) & / & / & / & $20(100.0)$ & $0(0)$ & / \\
\hline Sputum AFB smear or culture positive, n (\%) & $35(89.7)$ & $8(28.6)$ & 0 & / & / & / \\
\hline $1+$ & $11(28.2)$ & $7(25.0)$ & 0 & / & / & / \\
\hline$>1+(2+, 3+, 4+)$ & $24(61.5)$ & $1(3.6)$ & 0 & / & / & / \\
\hline Cavity, n (\%) & $19(48.7)$ & $1(3.6)$ & 0 & / & / & / \\
\hline \multicolumn{7}{|l|}{ M. tb detection in pleural effusion } \\
\hline Confirmed TBP by pleural biopsy, n (\%) & / & $8(28.6)$ & 0 & / & / & / \\
\hline AFB smear or culture positive, n (\%) & / & $3(10.7)$ & 0 & / & / & / \\
\hline \multicolumn{7}{|l|}{ anti-TB therapy status at enrollment } \\
\hline Naive, n (\%) & $8(20.5)$ & $23(82.1)$ & / & / & / & / \\
\hline Days on anti-TB, median (IQR) & $2(1-7)$ & $9(5-12)$ & / & / & / & / \\
\hline
\end{tabular}

Variables are shown as medians and IQRs. $X^{2}$ test was used for categorical data, and Kruskal-Wallis test was used for continuous data ATB active tuberculosis, PTB pulmonary tuberculosis, TBP tuberculous pleurisy, MPE malignant pleural effusion, $L T B I$ latent tuberculosis infection, $H C$ healthy control, IQR interquartile range, BCG bacillus Calmette-Guerin, IGRA interferon- $\gamma$ release assay, $A F B$ acid-fast bacilli 
$\mathbf{a}$

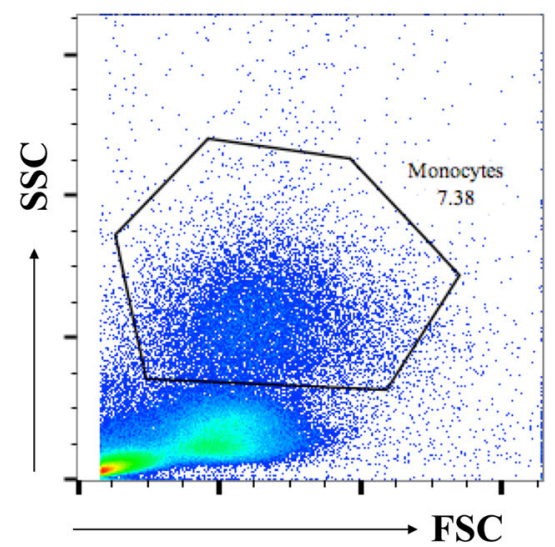

c

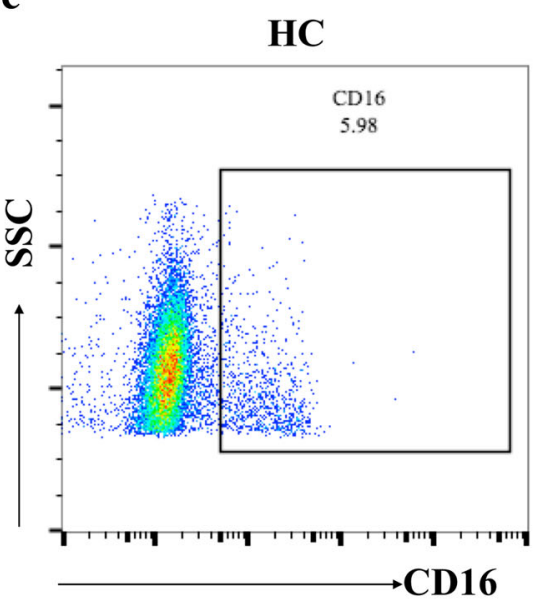

b

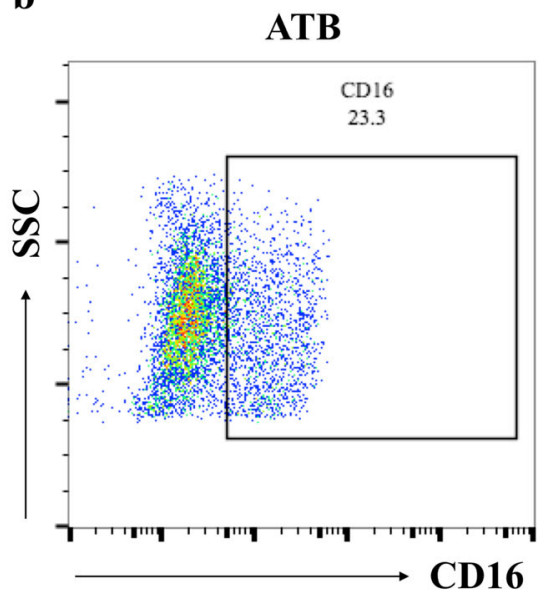

d

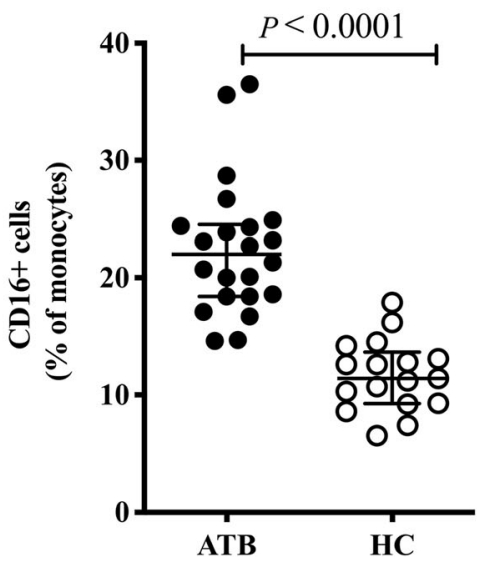

Fig. 1 Expression of CD16 on monocytes in ATB and HC groups. a Monocytes were gated based on FSC and SSC dot plots. b Representative monocyte-gated dot plots of CD16 expression in ATB patients. c Representative monocyte-gated dot plots of CD16 expression in HC. $\mathbf{d}$ Percentages of $\mathrm{CD}_{16} 6^{+}$monocytes were compared between the ATB and HC groups. Data are expressed as median with IQR and are analyzed using Mann-Whitney test. ATB, active tuberculosis; HC, healthy control; FSC, forward scatter; SSC, side scatter; IQR, interquartile range

higher percentages of $\mathrm{CD} 14^{+} \mathrm{CD}^{+} 6^{+}(15.7 \%$ vs $7.8 \%, P<$ $0.0001)$ and $\mathrm{CD}_{14}{ }^{-} \mathrm{CD}_{16}{ }^{+}(5.3 \%$ vs $2.5 \%, P=0.0011)$ monocyte subsets than the HC group (Fig. 2a and b). Notably, in this study, the $\mathrm{CD} 14^{+} \mathrm{CD} 16^{-}$monocytes subset, considered as the precursor of M1 macrophages, was significantly reduced in patients with ATB compared with the HC group (51.0\% vs $70.4 \%, P=0.0110$ ) (Fig. $2 \mathrm{a}$ and $\mathrm{b}$ ).

Higher expression of scavenger receptor CD163 on CD16 ${ }^{+}$ monocytes in active tuberculosis patients

Previously, the scavenger receptor CD163 was reported as a major biomarker of M2 macrophages [3], which play an anti-inflammatory role in tuberculosis infection. Thus, we hypothesized that CD163 was upregulated on the CD $14^{-}$CD $16^{+}$subset, namely the precursor of M2 macrophages. To test this, we analyzed the frequency of CD163expressing $\mathrm{CD}_{16}{ }^{+}$monocytes including $\mathrm{CD} 14^{+} \mathrm{CD} 16^{+}$and
$\mathrm{CD} 14^{-} \mathrm{CD} 16^{+}$subsets. As expected, ATB patients displayed a higher frequency of $\mathrm{CD} 163^{+}$cells in $\mathrm{CD}^{+} 6^{+}$ monocytes compared with the HC group (40.4\% vs $11.3 \%$, $P<0.0001$, Fig. 3a and b), in both CD14 ${ }^{+}(45.7 \%$ vs $11.3 \%$, $P<0.0001$, Fig. $3 \mathrm{a}$ and $\mathrm{c})$ and $\mathrm{CD}^{-} 4^{-}(7.8 \%$ vs $4.1 \%, P=$ 0.0100, Fig. 3a and c) subpopulations. Moreover, in ATB patients, we found that CD163 was mainly expressed on the $\mathrm{CD} 14^{+} \mathrm{CD} 16^{+}$rather than the $\mathrm{CD} 14^{-} \mathrm{CD} 16^{+}$subset (45.7\% vs $7.8 \%, P<0.0001$, Fig. 3 c).

Soluble CD163 levels in plasma and pleural effusion and the value of sCD163 in diagnosing tuberculosis

To our knowledge, scavenger receptor CD163 can be expressed on the surface of monocytes and macrophages, as well as in a soluble form in plasma. We thus determined the levels of sCD163 in the plasma of populations with different tuberculosis infection statuses. We 




observed significantly high levels of sCD163 both in ATB and LTBI groups, compared with those in the HC group $(1329.0 \mathrm{ng} / \mathrm{ml}$ vs $844.3 \mathrm{ng} / \mathrm{ml}, P=0.0004 ; 1304.0$ $\mathrm{ng} / \mathrm{ml}$ vs $844.3 \mathrm{ng} / \mathrm{ml}, P=0.0216$; Fig. 4a). However, there was no significant difference between the ATB and LTBI groups $(1329.0 \mathrm{ng} / \mathrm{ml}$ vs $1304.0 \mathrm{ng} / \mathrm{ml}, P=0.5045)$
(Fig. 4a). ROC analysis was also performed to evaluate the potential value of sCD163 in plasma for the differential diagnosis of PTB and HC. The area under ROC curve (AUC) was 0.7698 with an optimal cut-off value of $1260 \mathrm{ng} / \mathrm{ml}$ (sensitivity: 58.97\%, specificity: 90.48\%, Fig. 4b). Considering that TBP is the most common 


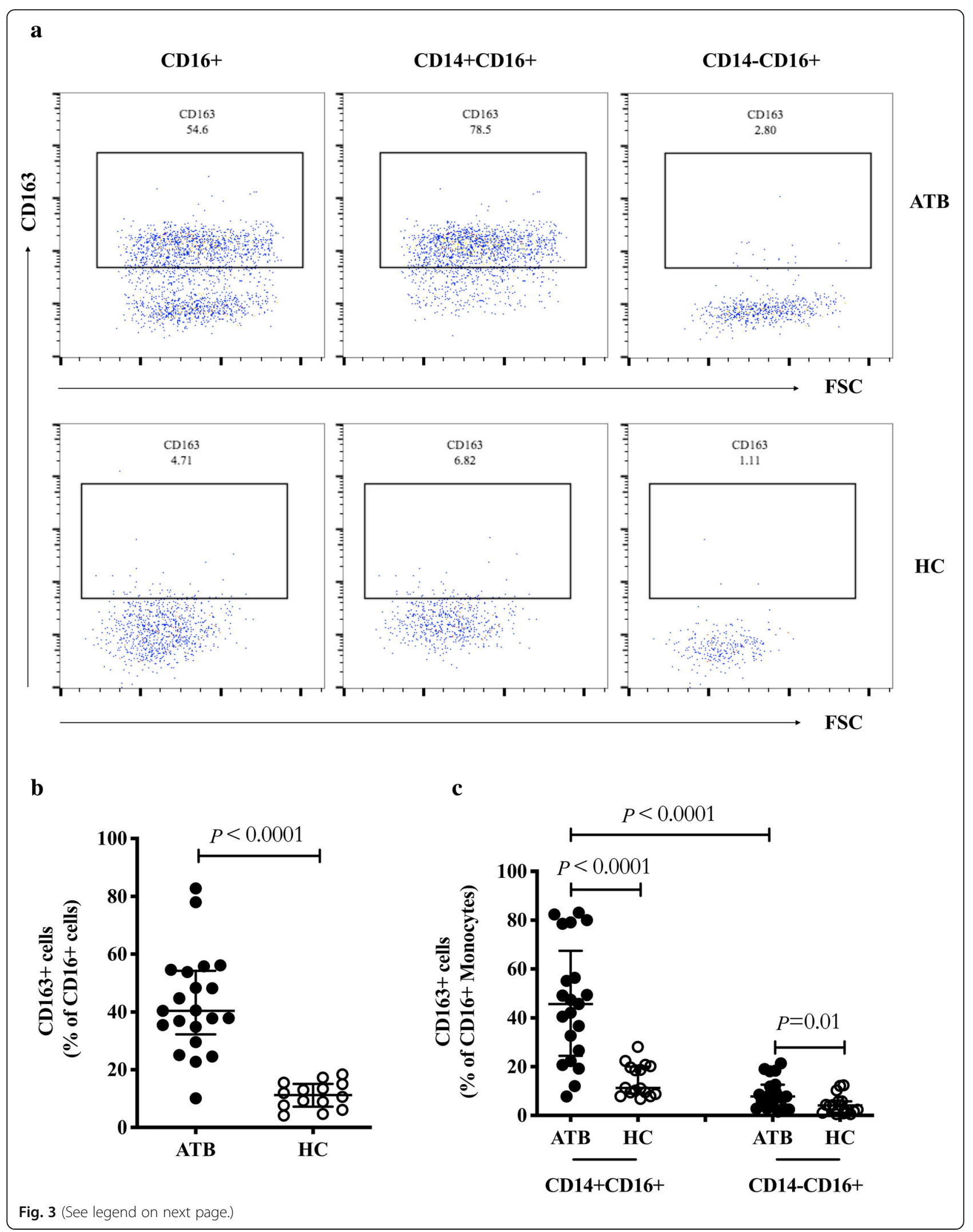


(See figure on previous page.)

Fig. 3 Expression of CD163 on CD16 monocytes in ATB and HC groups. a Representative dot plots of CD163 expression on CD16 (left), $\mathrm{CD} 14^{+} \mathrm{CD} 16^{+}$(center), and CD14-CD16 (right) monocytes in the ATB and HC groups. b-c Percentages of CD $163^{+}$cells among CD $16^{+}(\mathbf{b})$, $\mathrm{CD} 14^{+} \mathrm{CD} 16^{+}$, and $\mathrm{CD} 14^{-} \mathrm{CD} 16^{+}$monocytes (c) were compared between the ATB and HC groups. Data are expressed as median with IQR and are analyzed using Mann-Whitney test. ATB, active tuberculosis; HC, healthy control; IQR, interquartile range

extra-pulmonary tuberculosis, we next examined the levels of sCD163 in the PE and plasma of patients with TBP. As expected, sCD163 levels in the lesion of TBP patients were almost twice those in plasma $(2116.0 \mathrm{ng} /$ $\mathrm{ml}$ vs $1236.0 \mathrm{ng} / \mathrm{ml}, P<0.0001$, Fig. $4 \mathrm{c})$, and a similar result was seen in patients with MPE $(1820.0 \mathrm{ng} / \mathrm{ml}$ vs $783.6 \mathrm{ng} / \mathrm{ml}, P<0.0001$, Fig. 4c). Notably, the levels of sCD163 in PE and plasma samples of patients with TBP were significantly higher than those in patients with MPE $(2116.0 \mathrm{ng} / \mathrm{ml}$ vs $1820.0 \mathrm{ng} / \mathrm{ml}, P<0.0001 ; 1236.0$ $\mathrm{ng} / \mathrm{ml}$ vs $783.6 \mathrm{ng} / \mathrm{ml}, P=0.0040$, Fig. $4 \mathrm{c}$ ). Therefore, we further evaluated the value of sCD163 in PE and plasma for the differential diagnosis of patients with TBP and MPE. ROC analysis showed that sCD163 levels in PE and plasma for diagnosing TBP exhibited an AUC of 0.8418 and 0.8136 , respectively, with a cut-off value of $1950.0 \mathrm{ng} / \mathrm{ml}$ (sensitivity: 64.29\%, specificity: 100.00\%, Fig. 4d) and $934.7 \mathrm{ng} / \mathrm{ml}$ (sensitivity: $77.27 \%$, specificity: $80.00 \%$, Fig. 4e), respectively.

Relationship between soluble CD163 levels in plasma and disease severity in patients with pulmonary tuberculosis According to the grade of AFB sputum culture, PTB patients were divided into two groups: $1+$ and $>1+(2+, 3+$, and $4+$ ). Interestingly, we observed that the increased level of sCD163 in plasma was strongly associated with increased $M . t b$ loads in sputum (1+ group: $1012.0 \mathrm{ng} /$ ml, > 1+ group: $1461.0 \mathrm{ng} / \mathrm{ml}, P=0.0117$, Fig. $5 \mathrm{a}$ and b). In addition, among patients with PTB, approximately 48.7\% ( $n=19)$ showed cavity formation (Table 1$)$. We further compared the sCD163 levels in plasma between cavity (+) and cavity (-) groups, and found an evident increase of sCD163 in cavity $(+)$ group compared with that in cavity (-) group $(1445.0 \mathrm{ng} / \mathrm{ml}$ vs $1075.0 \mathrm{ng} / \mathrm{ml}$, $P=0.0242$, Fig. 5c). In Fig. 5c, the cut-off value of 1260 $\mathrm{ng} / \mathrm{ml}$ for the diagnosis of PTB was used as a threshold between sCD163 high (filled) and low (unfilled). Importantly, high sCD163 expression accounted for 16/19 $(84.2 \%)$ in the cavity (+) group, but $7 / 20(35.0 \%)$ in the cavity (-) group. These findings suggest a potential role of plasma sCD163 in predicting PTB disease severity.

\section{sCD163 levels in plasma decreased at different time points during anti-TB therapy}

We have previously found that increased plasma sCD163 levels were associated with disease severity (Fig. 5b and c). Next, we followed up with plasma samples of patients with
TBP at month 6 and month 9 during effective anti-TB treatment. As expected, the results demonstrated that sCD163 levels in plasma were decreased significantly at various time points during anti-TB therapy, especially at month 9 of treatment, compared with that at the baseline $(1180.0 \mathrm{ng} / \mathrm{ml}$ vs $685.9 \mathrm{ng} / \mathrm{ml}, P=0.0011$, Fig. 6). Furthermore, compared to the sCD163 levels during treatment for 6 months, they further decreased at month 9 of treatment (928.4 vs $685.9 \mathrm{ng} / \mathrm{ml}, P=0.0447$, Fig. 6). Therefore, changes in sCD163 levels might reflect changes in the burden of $M$. $t b$, which support the observations in Fig. 5.

\section{Discussion}

In this study, we compared three major monocytes subpopulations between PTB patients and HCs, and found an increased frequency of $\mathrm{CD} 14^{+} \mathrm{CD} 16^{+}$and $\mathrm{CD} 14^{-} \mathrm{CD} 16^{+}$ monocytes and a decreased percentage of $\mathrm{CD} 14^{+} \mathrm{CD} 16^{-}$ monocytes in patients with PTB. Currently, several studies have shown that changes in the profile of monocyte subsets during $M . t b$ infection indicate bacterial persistence [14-16]. A previous study has similarly demonstrated that an increased proportion of $\mathrm{CD} 14^{+} \mathrm{CD} 16^{+}$monocytes was associated with mortality in HIV-coinfected TB patients [17]. M. $t b$ infection has been shown to induce expansion of peripheral blood $\mathrm{CD} 16^{+}$monocytes spontaneously undergoing late apoptosis [18]. However, in the context of $\mathrm{TB}, \mathrm{CD}^{-} 6^{-}$(classical) monocytes as the dominant innate immune cells against $\mathrm{TB}$ contribute to the restriction of $M$. $t b$ growth by rapid migration to infection sites and high production of reactive oxygen species (ROS) [16]. $\mathrm{CD}_{14}{ }^{+} \mathrm{CD} 16^{+}$monocytes exhibited higher phagocytic activity and lower antigen presentation compared to the $\mathrm{CD} 14^{-} \mathrm{CD} 16^{+}$monocytes; additionally, this population was a major source of the immunosuppressive cytokine IL-10 [19]. Therefore, according to the characteristics of the above different monocyte subsets, our results indicated that an increase in non-classical $\left(\mathrm{CD} 14^{-} \mathrm{CD} 16^{+}\right)$and intermediate $\left(\mathrm{CD} 14^{+} \mathrm{CD} 16^{+}\right)$monocytes plus a decrease in classical $\left(\mathrm{CD} 14^{+} \mathrm{CD} 16^{-}\right)$monocytes might lead to the dissemination of $M . t b$ infection and be involved in the immunological pathogenesis of TB.

CD163 is considered a specific marker of M2 macrophages [20]. We explored the expression of CD163 on different $\mathrm{CD}_{16}{ }^{+}$monocytes subsets based on their CD14 expression. Surprisingly, scavenger receptor CD163 was mainly expressed on intermediate rather than non-classical monocytes, with a higher expression in PTB patients than 


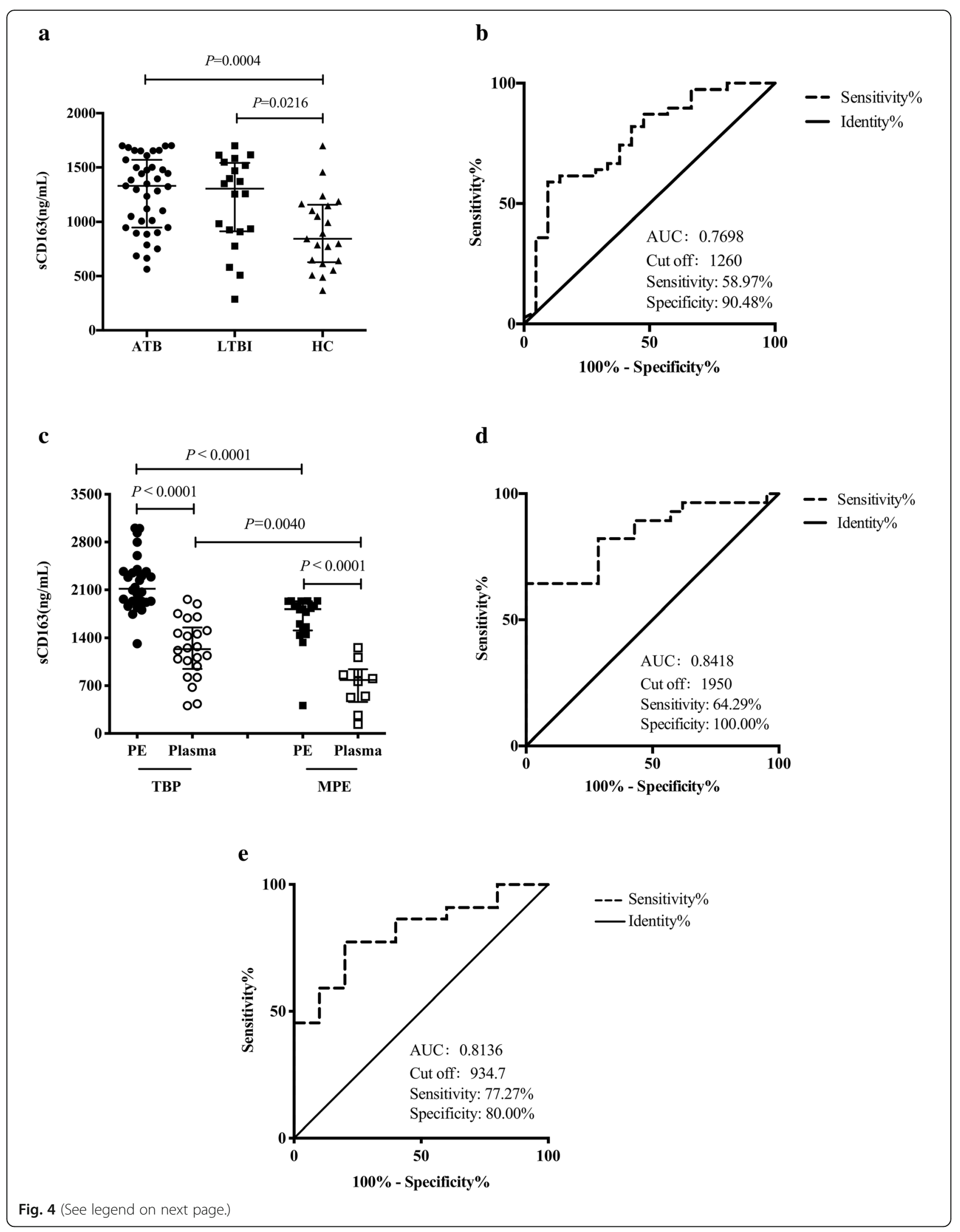


(See figure on previous page.)

Fig. 4 Levels of sCD163 in plasma and pleural effusion of individuals. a Expression of sCD163 in plasma of populations with different tuberculosis infection statuses. $\mathbf{b}$ ROC analysis of SCD163 in plasma for the differential diagnosis of PTB and HC. c Expression of SCD163 in the plasma and PE of TBP and MPE patients. d-e ROC analysis of SCD163 in PE (d) and in the plasma (e) for the differential diagnosis of TBP and MPE. Data are expressed as median with IQR and are analyzed using Mann-Whitney test. PTB, pulmonary tuberculosis; LTBI, latent tuberculosis infection; HC, healthy control; AUC, area under ROC curve; TBP, tuberculous pleurisy; MPE, malignant pleural effusion; PE, pleural effusion; IQR, interquartile range

$\mathbf{a}$
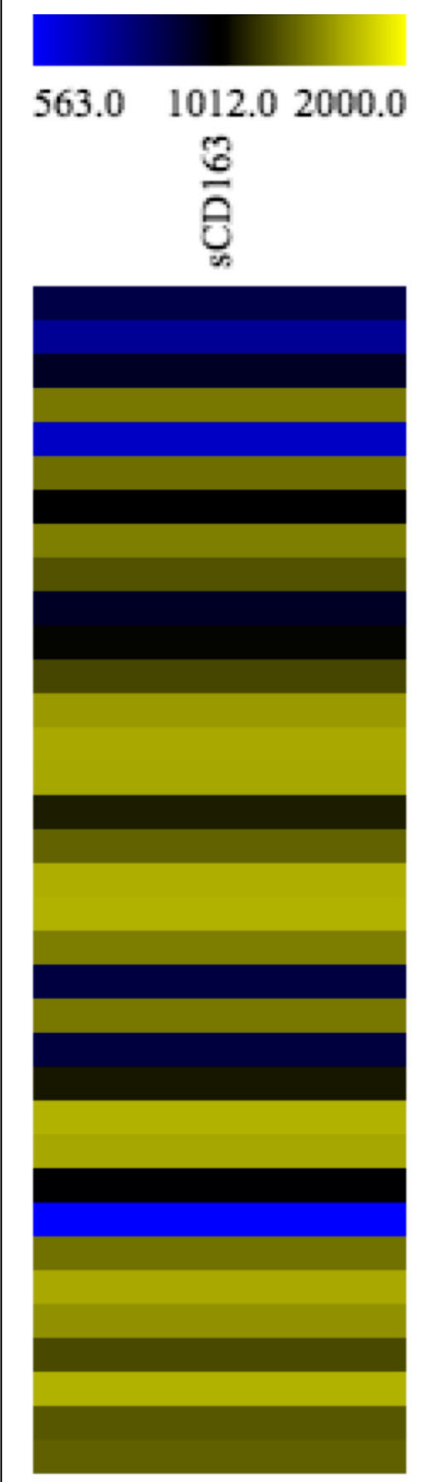

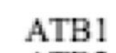

ATB2

ATB3

ATB4

ATB5

ATB6

ATB7

ATB8

ATB9

ATB 10

ATB 11

ATB 12

ATB 13

ATB 14

ATB15

ATB 16

ATB 17

ATB 18

ATB 19

ATB20

ATB21

ATB 22

ATB23

ATB24

ATB25

ATB26

ATB27

ATB 28

ATB29

ATB30

ATB31

ATB32

ATB33

ATB34

ATB35 b

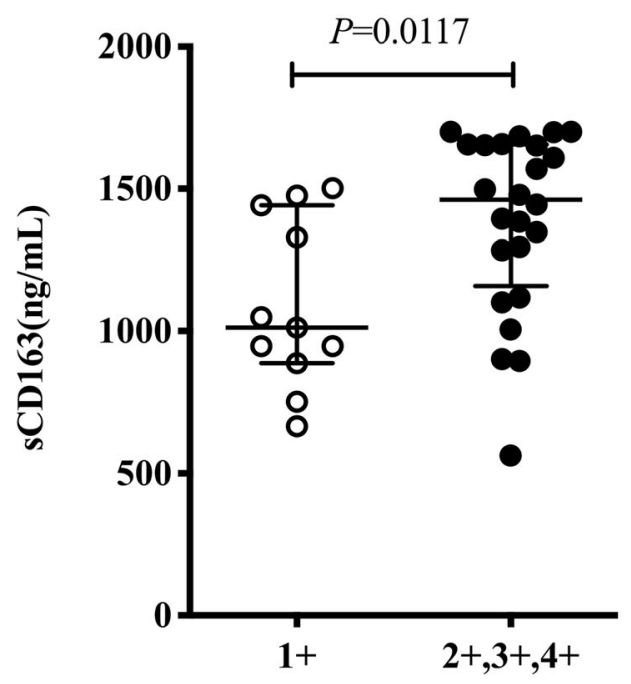

AFB sputum culture grade

c

$2+$

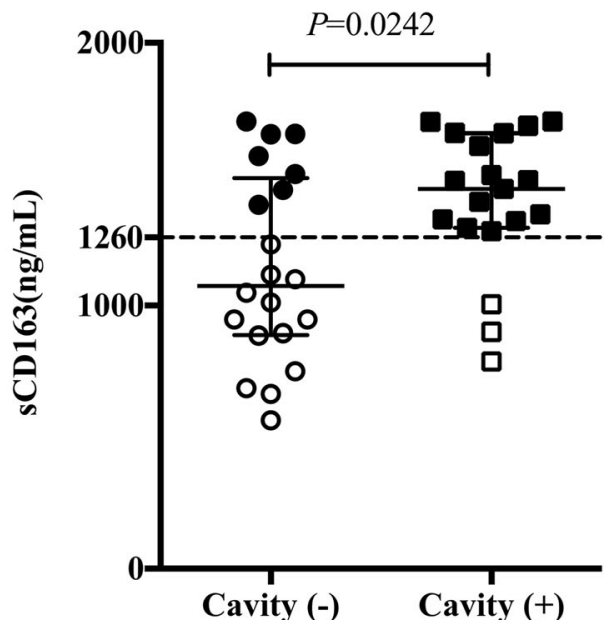

Fig. 5 Relationship between sCD163 levels in plasma and disease severity. a Heat map of plasma sCD163 levels in patients with AFB sputum culture-positive PTB. b Plasma sCD163 levels were compared between 1+ and > 1+ groups. c Plasma sCD163 levels were compared between cavity (-) and cavity (+) groups, the cut-off value of $1260 \mathrm{ng} / \mathrm{ml}$ (dotted line) for the diagnosis of PTB was used as the threshold between sCD163 high (filled) and low (unfilled). Data are expressed as median with IQR and are analyzed using Mann-Whitney test. PTB, pulmonary tuberculosis; ATB, active tuberculosis; AFB, acid-fast bacilli; IQR, interquartile range 




Fig. 6 sCD163 levels in plasma were decreased in response to effective anti-TB treatment. Data are expressed as median and extremum, and are analyzed using Mann-Whitney test. TB, tuberculosis

that in HCs. Cougoule et al. also observed M2 markers including CD163 and CD206 augmented in the CD16 ${ }^{+}$ subset compared to the $\mathrm{CD} 16^{-}$population, but they did not detect the expression of M2 markers in intermediate monocytes [12]. Indeed, they demonstrated that monocytes differentiated towards M2-like macrophages $\left(\mathrm{CD} 16^{+} \mathrm{CD} 163^{+} \mathrm{MerTK}^{+}\right)$depending on the IL10/STAT3 signaling pathway in the context of TB, and that this phenotype rendered the host permissive to intracellular $M$. $t b$ growth and an impaired ability to activate the Th1 immune response [12]. Furthermore, $\mathrm{CD}_{163}{ }^{+}$monocytes can secrete both pro- and antiinflammatory cytokines such as TNF- $\alpha$ and IL-4 during Leishmania and hepatitis $\mathrm{C}$ infection, which might interfere in instructing $\mathrm{T}$ cells and inhibit the killing of intracellular pathogens [21]. Thus, our findings indicated that high expression of CD163 on $\mathrm{CD}^{+} 6^{+}$monocytes in PTB patients might be involved in $M . t b$ infection.

Additionally, sCD163 has been recently considered a novel soluble biomarker of monocyte/macrophage activation in pathological conditions [22-24]. Consistent with the membrane CD163 expression, our results show increased levels of sCD163 in ATB patients, which were particularly higher in $\mathrm{PE}$ than in plasma, suggesting that $\mathrm{CD}_{163}{ }^{+}$monocytes might migrate to the site of infection in order to play an immunomodulatory role. This observation was consistent with the findings in patients with TB-associated immune reconstitution inflammatory syndrome (TB-IRIS), who exhibited high levels of sCD163 before anti-retroviral therapy (ART) and a worse prognosis
[25]. Our findings revealed sCD163 as a potential biomarker in the diagnosis of ATB with a high specificity and low sensitivity, allowing the distinction of TBP from MPE. With an optimal cut-off value of $1950.0 \mathrm{ng} / \mathrm{ml}$ and $934.7 \mathrm{ng} / \mathrm{ml}$, respectively, the sCD163 levels in PE and plasma showed AUCs of 0.8418 and 0.8136 in the diagnosis of TBP, respectively. Regrettably, sCD163 levels in plasma could not differentiate ATB from LTBI, whereas they could be used to distinguish ATB from HC with an AUC of 0.7698. Similarly, ROC analysis was conducted in TB patients and healthy subjects, showing a AUC of 0.78 , but the differences between tuberculous and malignant PE were not analyzed in this study [12]. Indeed, many biomarkers such as adenosine deaminase (ADA), lactate dehydrogenase (LDH), and IGRA, have been investigated to distinguish TBP from MPE patients, with a large range variation in sensitivity and specificity [26-29]. Based on our results, the sCD163 levels in PE and plasma could be a useful biomarker for the diagnosis of TBP.

More importantly, we found a strong association between high sCD163 levels and TB disease severity in the present study. First, the increased levels of sCD163 in plasma were strongly linked to increased $M . t b$ loads in sputum. Next, in the cavity (+) group, the proportion of high sCD163 (> $1260 \mathrm{ng} / \mathrm{ml}$ ) expression (84.2\%) was more than twice as large as that in the cavity $(-)$ group (35.0\%). Moreover, after effective anti-TB therapy, plasma sCD163 levels in patients with TBP were decreased significantly. A prospective cohort study followed up 113 verified TB patients, and demonstrated an association between high sCD163 levels (>3950 ng/ml) and increased mortality [30]. Actually, the serum levels of sCD163 in PTB patients were restored to normal levels after 12 months of anti-TB treatment [12]. These findings suggest that $\mathrm{sCD} 163$ might be a predictive biomarker for $\mathrm{TB}$ prognosis.

\section{Conclusions}

Taken together, our study indicates an increased frequency of $\mathrm{CD} 14^{+} \mathrm{CD} 16^{+}$and $\mathrm{CD} 14^{-} \mathrm{CD} 16^{+}$monocytes and a decreased $\mathrm{CD} 14^{+} \mathrm{CD} 16^{-}$population in PTB patients. Both membrane and soluble CD163 are markers of monocyte/macrophage activation, which were increased in patients with ATB, especially in pleural effusions. Furthermore, sCD163 can be used to distinguish TBP from MPE patients with a high specificity. Importantly, there was a strong association between the high sCD163 levels and TB disease severity. We also followed up plasma samples of TBP patients and observed a significant decrease in plasma sCD163 after effective anti-TB treatment. Therefore, CD163 may offer a new insight in the diagnosis and prognosis of TB patients. 


\section{Abbreviations}

AFB: Acid-fast bacilli; ELISA: Enzyme linked immunosorbent assay; HC: Healthy control; IGRA: Interferon- $\gamma$ release assay; LTBI: Latent tuberculosis infection; M. tb: Mycobacterium tuberculosis; MPE: Malignant pleural effusion; PBMCs: Peripheral blood mononuclear cells; PE: Pleural effusion; PTB: Pulmonary tuberculosis; TBP: Tuberculous pleurisy

\section{Acknowledgements}

We express sincere thanks to all the enrolled participants of this study.

\section{Authors' contributions}

LS conceived and designed the study. QL performed the experiments and drafted the manuscript. QO, YG, QR and WZ contributed as clinicians. HC, YL and $Y X$ analyzed the data. All authors read and approved the final version of the manuscript.

\section{Funding}

The present study was financially supported by the National Natural Science Foundation of China (No. 81501359 and No. 81671553), Wuxi Science and Technology Development Fund Project (CSE31N1713) and Wuxi Health and Family Planning Committee Youth Project (Q201765). The funding body had no role in the design of the study and collection, analysis, and interpretation of data and in writing the manuscript.

\section{Availability of data and materials}

The datasets generated and/or analyzed during the current study are not publicly available due to anonymity policy issues but are available from the corresponding author on reasonable request.

\section{Ethics approval and consent to participate}

This study was approved by the Ethics committee of Huashan Hospital, Fudan University. Written informed consent was obtained from all participants.

\section{Consent for publication}

Not applicable.

\section{Competing interests}

The authors declare that they have no competing interests.

\section{Author details}

'Department of Infectious Diseases, Huashan Hospital, Fudan University, 12 Wulumuqi Zhong Road, Shanghai 200040, China. ${ }^{2}$ Department of Pulmonary Diseases, Wuxi Infectious Diseases Hospital, Wuxi 214005, China. ${ }^{3}$ Key Laboratory of Medical Molecular Virology, Ministry of Education and Health, Shanghai Medical College, and Institutes of Biomedical Science, Fudan University, Shanghai 200032, China. ${ }^{4}$ State Key Laboratory of Genetic Engineering, School of Life Science, Fudan University, Shanghai 200438, China. ${ }^{5}$ National Clinical Research Center for Aging and Medicine, Huashan Hospital, Fudan University, Shanghai 200040, China.

Received: 12 May 2019 Accepted: 30 September 2019

\section{Published online: 28 November 2019}

\section{References}

1. Guilliams M, Ginhoux F, Jakubzick C, Naik SH, Onai N, Schraml BU, Segura E, Tussiwand R, Yona S. Dendritic cells, monocytes and macrophages: a unified nomenclature based on ontogeny. Nat Rev Immunol. 2014;14(8):571-8.

2. Benoit M, Desnues $B$, Mege JL. Macrophage polarization in bacterial infections. J Immunol. 2008;181(6):3733-9.

3. Lugo-Villarino G, Verollet C, Maridonneau-Parini I, Neyrolles O. Macrophage polarization: convergence point targeted by mycobacterium tuberculosis and HIV. Front Immunol. 2011;2:43.

4. Flynn JL, Chan J, Lin PL. Macrophages and control of granulomatous inflammation in tuberculosis. Mucosal Immunol. 2011;4(3):271-8.

5. Passlick B, Flieger D, Ziegler-Heitbrock HW. Identification and characterization of a novel monocyte subpopulation in human peripheral blood. Blood. 1989:74(7):2527-34.

6. Wong KL, Yeap WH, Tai JJ, Ong SM, Dang TM, Wong SC. The three human monocyte subsets: implications for health and disease. Immunol Res. 2012 53(1-3):41-57.
7. Ziegler-Heitbrock L, Ancuta P, Crowe S, Dalod M, Grau V, Hart DN, Leenen PJ, Liu YJ, MacPherson G, Randolph GJ, et al. Nomenclature of monocytes and dendritic cells in blood. Blood. 2010;116(16):e74-80.

8. Sampath P, Moideen K, Ranganathan UD, Bethunaickan R. Monocyte subsets: phenotypes and function in tuberculosis infection. Front Immunol. 2018:9:1726.

9. Redente EF, Higgins DM, Dwyer-Nield LD, Orme IM, Gonzalez-Juarrero M, Malkinson AM. Differential polarization of alveolar macrophages and bone marrow-derived monocytes following chemically and pathogen-induced chronic lung inflammation. J Leukoc Biol. 2010;88(1):159-68.

10. Dorhoi A, Reece ST, Kaufmann SH. For better or for worse: the immune response against mycobacterium tuberculosis balances pathology and protection. Immunol Rev. 2011:240(1):235-51.

11. Canton J, Neculai D, Grinstein S. Scavenger receptors in homeostasis and immunity. Nat Rev Immunol. 2013;13(9):621-34.

12. Lastrucci C, Benard A, Balboa L, Pingris K, Souriant S, Poincloux R, Al Saati T, Rasolofo V, Gonzalez-Montaner P, Inwentarz S, et al. Tuberculosis is associated with expansion of a motile, permissive and immunomodulatory CD16(+) monocyte population via the IL-10/STAT3 axis. Cell Res. 2015; 25(12):1333-51.

13. Shao L, Zhang W, Zhang S, Chen CY, Jiang W, Xu Y, Meng C, Weng X, Chen $Z W$. Potent immune responses of Ag-specific Vgamma2Vdelta2 $+T$ cells and CD8+ T cells associated with latent stage of Mycobacterium tuberculosis coinfection in HIV-1-infected humans. AIDS. 2008;22(17):2241-50.

14. Sanchez MD, Garcia Y, Montes C, Paris SC, Rojas M, Barrera LF, Arias MA, Garcia LF. Functional and phenotypic changes in monocytes from patients with tuberculosis are reversed with treatment. Microbes Infect. 2006;8(9-10): 2492-500

15. Balboa L, Romero MM, Basile Jl, Sabio y Garcia CA, Schierloh P, Yokobori N, Geffner L, Musella RM, Castagnino J, Abbate E, et al. Paradoxical role of CD16+CCR2+CCR5+ monocytes in tuberculosis: efficient APC in pleural effusion but also mark disease severity in blood. J Leukoc Biol. 2011;90(1):69-75.

16. Balboa L, Barrios-Payan J, Gonzalez-Dominguez E, Lastrucci C, Lugo-Villarino G, Mata-Espinoza D, Schierloh P, Kviatcovsky D, Neyrolles O, MaridonneauParini I, et al. Diverging biological roles among human monocyte subsets in the context of tuberculosis infection. Clin Sci (Lond). 2015;129(4):319-30.

17. Janssen S, Schutz C, Ward A, Nemes E, Wilkinson KA, Scriven J, Huson MA, Aben N, Maartens G, Burton R, et al. Mortality in severe human immunodeficiency virus-tuberculosis associates with innate immune activation and dysfunction of monocytes. Clin Infect Dis. 2017;65(1):73-82.

18. Castano D, Garcia LF, Rojas M. Increased frequency and cell death of CD16+ monocytes with mycobacterium tuberculosis infection. Tuberculosis. 2011; 91(5):348-60.

19. Skrzeczynska-Moncznik J, Bzowska M, Loseke S, Grage-Griebenow E, Zembala M, Pryjma J. Peripheral blood CD14high CD16+ monocytes are main producers of IL-10. Scand J Immunol. 2008;67(2):152-9.

20. Buechler C, Ritter M, Orso E, Langmann T, Klucken J, Schmitz G. Regulation of scavenger receptor CD163 expression in human monocytes and macrophages by pro- and antiinflammatory stimuli. J Leukoc Biol. 2000; 67(1):97-103.

21. Silva RL, Santos MB, Almeida PL, Barros TS, Magalhaes L, Cazzaniga RA, Souza PR, Luz NF, Franca-Costa J, Borges VM, et al. sCD163 levels as a biomarker of disease severity in leprosy and visceral leishmaniasis. PLoS Negl Trop Dis. 2017;11(3):e0005486.

22. Etzerodt A, Moestrup SK. CD163 and inflammation: biological, diagnostic, and therapeutic aspects. Antioxid Redox Signal. 2013;18(17):2352-63.

23. Fabriek BO, van Bruggen R, Deng DM, Ligtenberg AJ, Nazmi K, Schornagel $K$, Vloet RP, Dijkstra CD, van den Berg TK. The macrophage scavenger receptor CD163 functions as an innate immune sensor for bacteria. Blood. 2009;113(4):887-92.

24. Kneidl J, Loffler B, Erat MC, Kalinka J, Peters G, Roth J, Barczyk K. Soluble CD163 promotes recognition, phagocytosis and killing of Staphylococcus aureus via binding of specific fibronectin peptides. Cell Microbiol. 2012; 14(6):914-36.

25. Andrade BB, Singh A, Narendran G, Schechter ME, Nayak K, Subramanian S, Anbalagan S, Jensen SM, Porter BO, Antonelli LR, et al. Mycobacterial antigen driven activation of CD14++CD16- monocytes is a predictor of tuberculosis-associated immune reconstitution inflammatory syndrome. PLoS Pathog. 2014:10(10):e1004433.

26. Jeon D. Tuberculous pleurisy: an update. Tuberc Respir Dis. 2014;76(4):153-9. 
27. Blakiston M, Chiu W, Wong C, Morpeth S, Taylor S. Diagnostic performance of pleural fluid adenosine deaminase for tuberculous pleural effusion in a low-incidence setting. J Clin Microbiol. 2018;56(8). https://doi.org/10.1128/ JCM.00258-18.

28. Ferreiro L, San Jose E, Valdes L. Tuberculous pleural effusion. Arch Bronconeumol. 2014;50(10):435-43.

29. Porcel JM, Azzopardi M, Koegelenberg CF, Maldonado F, Rahman NM, Lee YC. The diagnosis of pleural effusions. Expert Rev Respir Med. 2015; 9(6):801-15.

30. Knudsen TB, Gustafson P, Kronborg G, Kristiansen TB, Moestrup SK, Nielsen JO, Gomes V, Aaby P, Lisse I, Moller HJ, et al. Predictive value of soluble haemoglobin scavenger receptor CD163 serum levels for survival in verified tuberculosis patients. Clin Microbiol Infect. 2005;11(9):730-5.

\section{Publisher's Note}

Springer Nature remains neutral with regard to jurisdictional claims in published maps and institutional affiliations.

Ready to submit your research? Choose BMC and benefit from:

- fast, convenient online submission

- thorough peer review by experienced researchers in your field

- rapid publication on acceptance

- support for research data, including large and complex data types

- gold Open Access which fosters wider collaboration and increased citations

- maximum visibility for your research: over $100 \mathrm{M}$ website views per year

At BMC, research is always in progress.

Learn more biomedcentral.com/submissions 